\title{
EXISTENCE OF POSITIVE SOLUTIONS FOR THE ONE-DIMENSION SINGULAR $P$-LAPLACIAN EQUATION WITH SIGN CHANGING NONLINEARITIES VIA THE METHOD OF UPPER AND LOWER SOLUTION *
}

\author{
HAISHEN LÜ๋ ${ }^{\dagger}$ DONAL O’REGAN ${ }^{\ddagger}$, AND RAVI P. AGARWAL $\S$
}

Abstract. A result concerning the existence of positive solutions for the Dirichlet boundary value problem $-\left(\varphi_{p}\left(u^{\prime}\right)\right)^{\prime}=f(t, u), t \in(0,1), u(0)=c>0$ and $u(1)=0$, is given in this paper. Here $f(t, y)$ may change sign and may be singular at $y=0$.

1. Introduction. This paper establishes a new result concerning the existence of nonnegative solutions for the Dirichlet boundary value problem

$$
\left\{\begin{array}{l}
-\left(\varphi_{p}\left(u^{\prime}\right)\right)^{\prime}=f(t, u), \quad t \in(0,1) \\
u(0)=c>0, u(1)=0
\end{array}\right.
$$

here $\varphi_{p}(x)=|x|^{p-2} x, p>1$. For $p=2$, the above problem models steady-state diffusion with reaction ( see [1] ) and many results have been obtained in the literature when $f(t, u) \leq 0$ or $f(t, u) \geq 0$, (see [2-4] and the references therein). However, very few results are available when $f(t, u)$ changes sign.

For $p \neq 2$, the above problem occurs in the study of the $n$-dimensional $p$-Laplace equation, non-Newtonian fluid theory and the turbulent flow of a gas in a porous medium [5].

2. Main Results. Consider the boundary value problem

$$
\left\{\begin{array}{l}
-\left(\varphi_{p}\left(u^{\prime}\right)\right)^{\prime}=F(t, u) \text { for all } t \in(0,1) \\
u(0)=a, u(1)=b
\end{array}\right.
$$

where $F: D \rightarrow R$ is continuous function and $D \subset(0,1) \times[0,+\infty)$.

Definition 2.1. Let $\alpha \in C([0,1], R) \cap C^{1}((0,1), R)$ and $\varphi_{p}\left(\alpha^{\prime}\right) \in$ $C^{1}((0,1), R)$. Now $\alpha$ is called a lower solution for problem $(2.1)$ if $(t, \alpha(t)) \in D$ for all $t \in(0,1)$ and

$$
\left\{\begin{array}{l}
-\left(\varphi_{p}\left(\alpha^{\prime}\right)\right)^{\prime} \leq F(t, \alpha(t)), \quad t \in(0,1) \\
\alpha(0) \leq a, \alpha(1) \leq b
\end{array}\right.
$$

Let $\beta \in C([0,1], R) \cap C^{1}((0,1), R)$ and $\varphi_{p}\left(\beta^{\prime}\right) \in C^{1}((0,1), R)$. Now $\beta$ is called an upper solution for problem $(2.1)$ if $(t, \beta(t)) \in D$ for all $t \in(0,1)$ and

$$
\left\{\begin{array}{l}
-\left(\varphi_{p}\left(\beta^{\prime}\right)\right)^{\prime} \geq F(t, \beta(t)), \quad t \in(0,1) \\
\beta(0) \geq a, \beta(1) \geq b
\end{array}\right.
$$

\footnotetext{
${ }^{*}$ Received August 27, 2003; accepted for publication November 26, 2003.

$\dagger$ Institute of Applied Mathematics, Academy of Mathematics and System Sciences, Chinese Academy of Science, Beijing 100080, China.

${ }^{\ddagger}$ Department of Mathematics, National University of Ireland, Galway, Ireland (donal.oregan@nuigalway.ie).

$\S$ Department of Mathematical Sciences, Florida Institute of Technology, Melbourne, FL 329016975, USA (agarwal@fit.edu).
} 
LEMma 2.1 [7]. Suppose $\alpha, \beta$ are lower and upper solution of problem (2.1) and assume the following conditions are satisfied:

$(H 1) \alpha(t) \leq \beta(t)$ for all $0 \leq t \leq 1$;

(H2) $D_{a \beta} \subseteq D$, here $D_{\alpha \beta}=\{(t, y) \mid 0<t<1, \alpha(t) \leq y \leq \beta(t)\}$;

(H3) there exists a continuous function $q \in C(0,1)$ such that

$$
|F(t, y)| \leq q(t), \quad \forall(t, y) \in D_{a \beta}
$$

and

$$
\int_{0}^{1} q(t) d t<+\infty
$$

Then the BVP $(2.1)$ has at least one solution $u \in C([0,1], R) \cap C^{1}((0,1), R)$ with $\varphi_{p}\left(u^{\prime}\right) \in C^{1}((0,1), R)$ such that

$$
\alpha(t) \leq u(t) \leq \beta(t), \quad 0 \leq t \leq 1
$$

THEOREM 2.1. Suppose the following conditions hold:

$(H 4) f:[0,1] \times(0,+\infty) \rightarrow(-\infty, \infty)$ is continuous and $\lim _{y \rightarrow 0^{+}} f(t, y)=-\infty$ uniformly on $[0,1]$;

$(H 5)$ there exist a constant $a \in(0, c]$ and a continuous function $g_{1}:(0, \infty) \rightarrow$ $(0, \infty)$ such that

$$
\begin{gathered}
f(t, u) \geq-g_{1}(u) \text { for } t \in[0,1], u>0, \int_{0}^{a} g_{1}(s) d s<+\infty \\
\text { and } \int_{0}^{a}\left(G_{1}(x)\right)^{-1 / p} d x>q^{1 / p} ;
\end{gathered}
$$

here $G_{1}(x)=\int_{0}^{x} g_{1}(s) d s, 0<x<a, q=\frac{p}{p-1}$;

$(H 6)$ there exists $b_{2}>c, b_{1} \in\left[0, b_{2}\right)$ and a continuous function $g_{2}:(0, \infty) \rightarrow$ $(0, \infty)$ such that

$$
f(t, u) \leq g_{2}(u) \text { for } t \in[0,1], u>0 \text { and } \int_{b_{1}}^{b_{2}}\left(G_{2}(x)\right)^{-1 / p} d x>q^{1 / p}
$$

here $G_{2}(x)=\int_{x}^{b_{2}} g_{2}(s) d s, b_{1}<x<b_{2}$.

Then (1.1) has at least one positive solution $u \in C^{1}[0,1] \cap C(0,1)$.

Proof. We first prove the following four Claims.

Claim 1. The problem

$$
\left\{\begin{array}{l}
\left(\varphi_{p}\left(\phi^{\prime}\right)\right)^{\prime}=g_{1}(\phi), t \in(0,1) \\
\phi(1)=0, \phi^{\prime}(1)=0, \phi(t)>0 \text { for } t \in[0,1)
\end{array}\right.
$$

has a unique solution $\phi \in C[0,1] \cap C^{1}(0,1)$ with

$$
0<\phi(t)<a, \quad \forall t \in[0,1) \text {. }
$$

In addition

$$
-\left(\varphi_{p}\left(\phi^{\prime}\right)\right)^{\prime} \leq f(t, \phi) \text { for } t \in(0,1) \text {. }
$$


Proof of Claim 1. From [9] we know that (2.2) has a unique positive solution. On the other hand, since $\left(\varphi_{p}\left(\phi^{\prime}\right)\right)^{\prime} \geq 0$ we have that $\phi^{\prime}$ is increasing. Using $\phi^{\prime}(1)=0$ we obtain $\phi^{\prime} \leq 0$.

Multiply both sides of (2.1) by $\phi^{\prime}$ and then integrate from $s$ to 1 , to obtain

$$
\int_{s}^{1}\left(\varphi_{p}\left(\phi^{\prime}(t)\right)\right)^{\prime} \phi^{\prime}(t) d t=\int_{s}^{1} g_{1}(\phi(t)) \phi^{\prime}(t) d t
$$

SO

$$
\int_{0}^{\varphi_{p}\left(\phi^{\prime}(s)\right)} \varphi_{p}^{-1}(x) d x=G_{1}(\phi(s))
$$

Now use

$$
\int_{0}^{u} \varphi_{p}^{-1}(s) d s=\frac{1}{q}|u|^{q},
$$

to obtain

$$
\frac{1}{q}\left|\phi^{\prime}\right|^{p}=G_{1}(\phi)
$$

Thus

$$
\phi^{\prime}=-q^{\frac{1}{p}}\left(G_{1}(\phi)\right)^{\frac{1}{p}},
$$

SO

$$
\int_{t}^{1} \frac{d \phi}{\left(G_{1}(\phi)\right)^{\frac{1}{p}}}=-q^{\frac{1}{p}}(1-t) \text { for } t \in[0,1] .
$$

Consequently

$$
\int_{0}^{\phi(t)}\left(G_{1}(x)\right)^{\frac{-1}{p}} d x=q^{\frac{1}{p}}(1-t) \text { for } t \in[0,1]
$$

Let

$$
H_{1}(u)=\int_{0}^{u}\left(G_{1}(x)\right)^{\frac{-1}{p}} d x .
$$

Then

$$
\phi(s)=H_{1}^{-1}\left(q^{\frac{1}{p}}(1-s)\right)
$$

is a solution of $(2.2)$. Now $\int_{0}^{\phi(0)}\left(G_{1}(x)\right)^{\frac{-1}{p}} d x=q^{\frac{1}{p}}$ and $\int_{0}^{a}\left(G_{1}(x)\right)^{\frac{-1}{p}} d x>q^{\frac{1}{p}}$ imply $0<\phi(0)<a$. Also since $\phi^{\prime} \leq 0$ we have

$$
\left(\varphi_{p}\left(\phi^{\prime}\right)\right)+f(t, \phi) \geq\left(\varphi_{p}\left(\phi^{\prime}\right)\right)^{\prime}-g_{1}(\phi)=0 \text { for } t \in(0,1) .
$$

Claim 2. The problem

$$
\left\{\begin{array}{l}
-\left(\varphi_{p}\left(\theta^{\prime}\right)\right)^{\prime}=g_{2}(\theta), t \in(0,1) \\
\theta(0)=b_{2}, \theta^{\prime}(0)=0, \theta(t)>0 \text { for } t \in(0,1]
\end{array}\right.
$$


has a unique solution $\theta \in C[0,1] \cap C^{1}(0,1)$ such that

$$
b_{1}<\theta(t)<b_{2}, \quad \forall t \in(0,1] \text {. }
$$

In addition

$$
-\left(\varphi_{p}\left(\theta^{\prime}\right)\right)^{\prime} \geq f(t, \theta) \text { for } t \in(0,1)
$$

Proof of Claim 2. From [9] we know that (2.4) has a unique positive solution. On the other hand since $-\left(\varphi_{p}\left(\theta^{\prime}\right)\right)^{\prime} \geq 0$ we have that $\theta^{\prime}$ is decreasing. Using $\theta^{\prime}(0)=0$ we obtain $\theta^{\prime} \leq 0$. Let

$$
H_{2}(x)=\int_{x}^{b_{2}}\left(G_{2}(s)\right)^{\frac{-1}{p}} d s, \quad 0<x<b_{2} .
$$

Argue as in Claim 1 to obtain

$$
\int_{\theta(t)}^{b_{2}}\left(G_{2}(x)\right)^{\frac{-1}{p}} d x=q^{\frac{1}{p}} t \text { for } t \in(0,1] .
$$

Then

$$
\theta(t)=H_{2}^{-1}\left(q^{\frac{1}{p}} t\right) \text { for } t \in(0,1]
$$

is a solution of $(2.4)$. Now since $\int_{\theta(1)}^{b_{2}}\left(G_{2}(x)\right)^{\frac{-1}{p}} d x=q^{\frac{1}{p}}$ and $\int_{b_{1}}^{b_{2}}\left(G_{2}(x)\right)^{\frac{-1}{p}} d x>q^{\frac{1}{p}}$ we have $b_{1}<\theta(1)<b_{2}$. Moreover

$$
\left(\varphi_{p}\left(\theta^{\prime}\right)\right)^{\prime}+f(t, \theta) \leq\left(\varphi_{p}\left(\theta^{\prime}\right)\right)^{\prime}+g_{2}(\theta)=0 \text { for } t \in(0,1) .
$$

Claim 3. $\phi(t)<\theta(t)$ for all $t \in(0,1)$.

Proof of Claim 3. If $a \leq b_{1}$ then $\phi(t)<a \leq b_{1}<\theta(t)$ for $t \in(0,1)$. We now consider the case $b_{1}<a$. We easily obtain that

$$
G_{1}(u)>0 \text { for } u \in(0, a), G_{2}(u)>0 \text { for } u \in\left(b_{1}, b_{2}\right)
$$

and

$$
q^{\frac{1}{p}}=\int_{0}^{\phi(t)}\left(G_{1}(x)\right)^{\frac{-1}{p}} d x+\int_{\theta(t)}^{b_{2}}\left(G_{2}(x)\right)^{\frac{-1}{p}} d x \text { for } t \in(0,1) .
$$

Let

$$
\Phi(u)=\int_{0}^{u}\left(G_{1}(x)\right)^{\frac{-1}{p}} d x+\int_{u}^{b_{2}}\left(G_{2}(x)\right)^{\frac{-1}{p}} d x \text { for } u \in\left[b_{1}, a\right] .
$$

It is obvious that $\Phi \in C\left[b_{1}, a\right] \cap C^{2}\left(b_{1}, a\right)$ with

$$
\Phi^{\prime}(u)=\left(G_{1}(u)\right)^{\frac{-1}{p}}-\left(G_{2}(u)\right)^{\frac{-1}{p}} \text { for } u \in\left(b_{1}, a\right),
$$

and

$$
-p \Phi^{\prime \prime}(u)=\left(G_{1}(u)\right)^{\frac{-1}{p}-1} g_{1}(u)+\left(G_{2}(u)\right)^{\frac{-1}{p}-1} g_{2}(u) \text { for } u \in\left(b_{1}, a\right) \text {. }
$$


If $\Phi^{\prime}\left(u_{0}\right)=0$ for $u_{0} \in[b, a]$, then $G_{1}\left(u_{0}\right)=G_{2}\left(u_{0}\right)>0$. On the other hand, $g_{1}\left(u_{0}\right)+g_{2}\left(u_{0}\right)>0$, so

$$
-p \Phi^{\prime \prime}\left(u_{0}\right)=\left(G_{1}\left(u_{0}\right)\right)^{\frac{-1}{p}-1}\left(g_{1}\left(u_{0}\right)+g_{2}\left(u_{0}\right)\right)>0 .
$$

Consequently, $\Phi$ has no locally minimum point in $\left(b_{1}, a\right)$. Notice

$$
\Phi\left(b_{1}\right)=\int_{0}^{b_{1}}\left(G_{1}(x)\right)^{\frac{-1}{p}} d x+\int_{b_{1}}^{b_{2}}\left(G_{2}(x)\right)^{\frac{-1}{p}} d x \geq \int_{b_{1}}^{b_{2}}\left(G_{2}(x)\right)^{\frac{-1}{p}} d x>q^{\frac{1}{p}} .
$$

Since $a<b_{2}$ we have

$$
\Phi(a)=\int_{0}^{a}\left(G_{1}(x)\right)^{\frac{-1}{p}} d x+\int_{a}^{b_{2}}\left(G_{2}(x)\right)^{\frac{-1}{p}} d x \geq \int_{0}^{a}\left(G_{1}(x)\right)^{\frac{-1}{p}} d x>q^{\frac{1}{p}} .
$$

Consequently

$$
\Phi(u)=\int_{0}^{u}\left(G_{1}(x)\right)^{\frac{-1}{p}} d x+\int_{u}^{b_{2}}\left(G_{2}(x)\right)^{\frac{-1}{p}} d x>q^{\frac{1}{p}} \text { for } u \in\left[b_{1}, a\right] .
$$

Suppose there exists $t_{0} \in(0,1)$ such that $\theta\left(t_{0}\right)<\phi\left(t_{0}\right)$. Then $b_{1}<\theta\left(t_{0}\right)<$ $\phi\left(t_{0}\right)<a$. By $(2.3)$ and $(2.6)$ we have

$$
\begin{aligned}
q^{\frac{1}{p}} & =\int_{0}^{\phi\left(t_{0}\right)}\left(G_{1}(x)\right)^{\frac{-1}{p}} d x+\int_{\theta\left(t_{0}\right)}^{\phi\left(t_{0}\right)}\left(G_{2}(x)\right)^{\frac{-1}{p}} d x+\int_{\phi\left(t_{0}\right)}^{b_{2}}\left(G_{2}(x)\right)^{\frac{-1}{p}} d x \\
& \geq \int_{0}^{\phi\left(t_{0}\right)}\left(G_{1}(x)\right)^{\frac{-1}{p}} d x+\int_{\phi\left(t_{0}\right)}^{b_{2}}\left(G_{2}(x)\right)^{\frac{-1}{p}} d x \\
& =\Phi\left(\phi\left(t_{0}\right)\right) \\
& >q^{\frac{1}{p}}(\text { see }(2.7)),
\end{aligned}
$$

a contradiction.

Claim 4. There exists $\eta \in C[0,1] \cap C^{1}(0,1)$ such that $\phi(t) \leq \eta(t) \leq \theta(t), \forall t \in$ $(0,1)$ and

$$
\left\{\begin{array}{l}
-\left(\varphi_{p}\left(\eta^{\prime}\right)\right)^{\prime} \geq f(t, \eta), \quad t \in(0,1) \\
\eta(0)=c, \eta(1)=0 .
\end{array}\right.
$$

Proof of Claim 4. Let $R=\min _{t \in[0,1]} \theta(t)>0$ and

$$
F(t, y)=\left\{\begin{array}{l}
f(t, y), \quad y \geq R \\
\max \{f(t, y), f(t, R)\}, \quad 0<y<R \\
f(t, R), \quad y=0
\end{array}\right.
$$

First we prove that $F:[0,1] \times[0, \infty) \rightarrow(-\infty, \infty)$ is continuous. By $(H 4)$, there exist $\delta, 0<\delta<R$, such that $f(t, y)<f(t, R)$ for all $(t, y) \in[0,1] \times(0, \delta]$. As a result

$$
F(t, y)=f(t, R) \text { for }(t, y) \in[0,1] \times(0, \delta],
$$

so $F:[0,1] \times[0, \infty) \rightarrow(-\infty, \infty)$ is continuous. 
By Claim 1 and Claim 2, we have

$$
\begin{aligned}
& -\left(\varphi_{p}\left(\theta^{\prime}(t)\right)\right)^{\prime}-F(t, \theta(t))=-\left(\varphi_{p}\left(\theta^{\prime}(t)\right)\right)^{\prime}-f(t, \theta(t)) \geq 0, \quad t \in(0,1) \\
& -\left(\varphi_{p}\left(\phi^{\prime}(t)\right)\right)^{\prime}-F(t, \phi(t)) \leq-\left(\varphi_{p}\left(\phi^{\prime}(t)\right)\right)^{\prime}-f(t, \phi(t)) \leq 0, \quad t \in(0,1)
\end{aligned}
$$

and

$$
0<\phi(0)<c \leq \theta(0), \phi(1)=0<\theta(1), 0<\phi(t)<\theta(t) \text { for } t \in(0,1) .
$$

From Lemma 2.1, we know the problem

$$
\left\{\begin{array}{l}
-\left(\varphi_{p}\left(\eta^{\prime}(t)\right)\right)^{\prime}=F(t, \eta(t)), \quad t \in(0,1) \\
\eta(0)=c, \quad \eta(1)=0
\end{array}\right.
$$

has a solution $\eta \in C[0,1] \cap C^{1}(0,1)$ with $\phi(t) \leq \eta(t) \leq \theta(t), \forall t \in(0,1)$. Since $F(t, y) \geq f(t, y),(t, y) \in(0,1) \times(0, \infty)$, we have $-\left(\varphi_{p}\left(\eta^{\prime}(t)\right)\right)^{\prime} \geq f(t, \eta(t))$ for all $t \in(0,1)$.

Proof of Theorem 2.1. For $n \in\{3,4, \cdots\}$, consider the problem

$$
\left\{\begin{array}{l}
\left(\varphi_{p}\left(z^{\prime}(t)\right)\right)^{\prime}-f(t, z(t))=0, \quad t \in\left(0, \frac{n-1}{n}\right) \\
z(0)=c, \quad z\left(\frac{n-1}{n}\right)=\eta\left(\frac{n-1}{n}\right)
\end{array}\right.
$$

From Claim 1 and Claim 4, we have

$$
\left\{\begin{array}{l}
-\left(\varphi_{p}\left(\eta^{\prime}\right)\right)^{\prime} \geq f(t, \eta), \quad t \in\left(0, \frac{n-1}{n}\right) \\
\eta(0)=c, \eta\left(\frac{n-1}{n}\right)=\eta\left(\frac{n-1}{n}\right)
\end{array}\right.
$$

and

$$
\left\{\begin{array}{l}
-\left(\varphi_{p}\left(\phi^{\prime}\right)\right)^{\prime} \leq f(t, \phi), \quad t \in\left(0, \frac{n-1}{n}\right) \\
\phi(0) \leq c, \phi\left(\frac{n-1}{n}\right) \leq \eta\left(\frac{n-1}{n}\right)
\end{array}\right.
$$

Then $\eta$ is an upper solution and $\phi$ is a lower solution of problem (2.8). On the other hand $0<\phi(t) \leq \eta(t), t \in\left[0,1-\frac{1}{n}\right]$ and $f:\left[0,1-\frac{1}{n}\right] \times D_{\phi \eta} \rightarrow(-\infty, \infty)$ is continuous. From Lemma 2.1, problem (2.8) has at least one solution $z_{n} \in$ $C\left(\left[0, \frac{n-1}{n}\right], R\right) \cap C^{1}\left(\left(0, \frac{n-1}{n}\right), R\right)$ and $\varphi_{p}\left(z_{n}^{\prime}\right) \in C^{1}\left(\left(0, \frac{n-1}{n}\right), R\right)$ such that

$$
\phi(t) \leq z_{n}(t) \leq \eta(t) \text { for } 0 \leq t \leq \frac{n-1}{n} .
$$

Fix $n_{0} \in\{3,4, \cdots\}$. Now lets look at the interval $\left[0,1-\frac{1}{n_{0}}\right]$. Let

$$
R_{n_{0}}=\sup \left\{|f(t, u)|: t \in\left[0,1-\frac{1}{n_{0}}\right] \text { and } u \in D_{\phi \eta}\right\} \text {. }
$$

The Mean Value Theorem implies that there exists $\tau \in\left(0,1-\frac{1}{n_{0}}\right)$ with $\left|z_{n}^{\prime}(\tau)\right| \leq$ $3 \sup _{[0,1]} \eta(t) \equiv L_{n_{0}}$. Hence for $t \in\left[0,1-\frac{1}{n_{0}}\right]$ we have

$$
\left|z_{n}^{\prime}(t)\right| \leq \varphi_{p}^{-1}\left(\left|z_{n}^{\prime}(\tau)\right|+\left|\int_{\tau}^{t}\left(\varphi_{p}\left(z_{n}^{\prime}\right)\right)^{\prime} d x\right|\right) \leq\left(\varphi_{p}\left(L_{n_{0}}\right)+R_{n_{0}}\right)^{\frac{1}{p-1}}
$$


where $\varphi_{p}^{-1}$ is an inverse function of $\varphi_{p}$.

As a result

$$
\left\{z_{n}\right\}_{n=n_{0}}^{\infty} \text { is bounded, equicontinuous family on }\left[0,1-\frac{1}{n_{0}}\right] .
$$

The Arzela-Ascoli theorem guarantees the existence of a subsequence $N_{n_{0}}$ of integers and a function $u_{n_{0}} \in C\left[0,1-\frac{1}{n_{0}}\right]$ with $z_{n}$ converging uniformly to $u_{n_{0}}$ on $\left[0,1-\frac{1}{n_{0}}\right]$ as $n \rightarrow \infty$ through $N_{n_{0}}$. Similarly

$$
\left\{z_{n}\right\}_{n=n_{0}}^{\infty} \text { is bounded, equicontinuous family on }\left[0,1-\frac{1}{n_{0}+1}\right] \text {, }
$$

so there is a subsequence $N_{n_{0}+1}$ of $N_{n_{0}}$ and a function $u_{n_{0}+1} \in C\left[0,1-\frac{1}{n_{0}+1}\right]$ with $z_{n}$ converging uniformly to $u_{n_{0}+1}$ on $\left[0,1-\frac{1}{n_{0}+1}\right]$ as $n \rightarrow \infty$ through $N_{n_{0}+1}$. Note $u_{n_{0}+1}=u_{n_{0}}$ on $\left[0,1-\frac{1}{n_{0}}\right]$ since $N_{n_{0}+1} \subseteq N_{n_{0}}$. Proceed inductively to obtain subsequence on integers

$$
N_{n_{0}} \supseteq N_{n_{0}+1} \supseteq \cdots \supseteq N_{k} \supseteq \cdots
$$

and functions

$$
u_{k} \in\left[0,1-\frac{1}{k}\right]
$$

with

$$
z_{n} \text { converging uniformly to } u_{k} \text { on }\left[0,1-\frac{1}{k}\right] \text { as } n \rightarrow \infty \text { through } N_{k}
$$

and

$$
u_{k+1}=u_{k} \text { on }\left[0,1-\frac{1}{k}\right] \text {. }
$$

Define a function $u:[0,1] \rightarrow[0, \infty)$ by $u(t)=u_{k}(t)$ on $\left[0,1-\frac{1}{k}\right]$ and $u(1)=0$. Notice $u$ is well defined and $\phi(t) \leq u(t) \leq \eta(t)$ for $t \in(0,1)$. Next fix $t \in[0,1)$ and let $m \in\left\{n_{0}, n_{0}+1, \cdots\right\}$ be such that $0 \leq t<1-\frac{1}{m}$. Let $N_{m}^{+}=\left\{n \in N_{m}: n \geq m\right\}$. Let $n \in N_{m}^{+}$and let $a=0, b=1-\frac{1}{m}$.

Define the operator, $L: C\left[a_{0}, b_{0}\right] \rightarrow C\left[a_{0}, b_{0}\right]$ by

$$
(L y)(t)=y\left(a_{0}\right)+\int_{a_{0}}^{t} \varphi_{p}^{-1}\left(A_{y}+\int_{s}^{b_{0}} q(\tau) f(\tau, y(\tau)) d \tau\right) d s
$$

where $A_{y}$ satisfies

$$
\int_{a_{0}}^{b_{0}} \varphi_{p}^{-1}\left(A_{y}+\int_{s}^{b_{0}} f(\tau, y(\tau)) d \tau\right) d s=y\left(b_{0}\right)-y\left(a_{0}\right) .
$$

Let $y_{n} \rightarrow y$ uniformly on $\left[a_{0}, b_{0}\right]$. As the proof in Theorem $2.4^{[5]}$, if we show $\lim _{n \rightarrow \infty} A_{y_{n}}=A$, then this together with $\varphi_{p}^{-1}$ continuous, implies that 
$L: C\left[a_{0}, b_{0}\right] \rightarrow C\left[a_{0}, b_{0}\right]$ is continuous. Associate $A_{y_{n}}$ with $y_{n}$ and notice

$$
\begin{aligned}
& \int_{a_{0}}^{b_{0}}\left(\varphi_{p}^{-1}\left(A_{y_{n}}+\int_{s}^{b_{0}} f(\tau, y(\tau)) d \tau\right)-\varphi_{p}^{-1}\left(A_{y}+\int_{s}^{b_{0}} f(\tau, y(\tau)) d \tau\right)\right) d s \\
= & y_{n}\left(b_{0}\right)-y_{n}\left(a_{0}\right)-y\left(b_{0}\right)+y\left(a_{0}\right) .
\end{aligned}
$$

The Mean Value Theorem for integrals implies that there exists $\eta_{n} \in[0,1]$ with

$$
\begin{aligned}
& \varphi_{p}^{-1}\left(A_{y_{n}}+\int_{\eta_{n}}^{b_{0}} f(\tau, y(\tau)) d \tau\right)-\varphi_{p}^{-1}\left(A_{y}+\int_{\eta_{n}}^{b_{0}} f(\tau, y(\tau)) d \tau\right) \\
= & \frac{y_{n}\left(b_{0}\right)-y_{n}\left(a_{0}\right)-y\left(b_{0}\right)+y\left(a_{0}\right)}{b_{0}-a_{0}},
\end{aligned}
$$

and since $y_{n} \rightarrow y$ uniformly on $\left[a_{0}, b_{0}\right]$ we have $\lim _{n \rightarrow \infty} A_{y_{n}}=A_{y}$.

Now since $z_{n}$ converges uniformly on $\left[a_{0}, b_{0}\right]$ to $u$ as $n \rightarrow \infty$ and $L z_{n}=z_{n}$ we obtain $L u=u$, i.e.

$$
-\left(\varphi_{p}\left(u^{\prime}(t)\right)\right)^{\prime}=f(t, u), a_{0} \leq t \leq b_{0} .
$$

We can do this argument for each $t \in(0,1)$ and so $-\left(\varphi_{p}\left(u^{\prime}(t)\right)\right)^{\prime}=f(t, u), \quad 0<t<$ 1. It remains to show $u$ is continuous at 1 .

Let $\varepsilon>0$ be given. Now since $0<\phi(t) \leq \eta(t), t \in(0,1)$ and $\phi(1)=\eta(1)=0$, there exists $\delta>0$ with

$$
0 \leq \phi(t) \leq \eta(t)<\frac{\varepsilon}{2} \text { for } t \in[1-\delta, 1] .
$$

This together with the fact that $\phi(t) \leq u_{n}(t) \leq \eta(t)$ for $t \in(0,1)$ implies that

$$
\phi(t) \leq u_{n}(t) \leq \eta(t)<\frac{\varepsilon}{2} \text { for } t \in[1-\delta, 1] .
$$

Consequently

$$
0 \leq \phi(t) \leq u(t) \leq \eta(t)<\frac{\varepsilon}{2} \text { for } t \in[1-\delta, 1]
$$

and so $u$ is continuous at 1 . Thus $u \in C[0,1] \cap C^{1}(0,1)$ and $u$ is a positive solution of (1.1). The proof of Theorem 2.1 is complete.

REMARK 2.1. The ideas in this section can be used to discuss the BVP

$$
\left\{\begin{array}{l}
-\left(\varphi_{p}\left(u^{\prime}\right)\right)^{\prime}=f(t, u), \quad t \in(0,1) \\
u(0)=0, u(1)=c>0 .
\end{array}\right.
$$

Only minor adjustments are needed, so we leave the details to the reader.

To illustrate the above ideas we consider the following problem

$$
\left\{\begin{array}{l}
-\left(\varphi_{p}\left(u^{\prime}\right)\right)^{\prime}=\lambda a(t)\left(u^{\beta}-\frac{1}{u^{\alpha}}\right) \text { for } t \in(0,1) \\
u(0)=c>0, u(1)=0
\end{array}\right.
$$

here $\lambda>0, a \in C[0,1]$ and $a(t)>0$ for $t \in[0,1], 0<\alpha<1$ and $0<\beta$.

Corollary 2.1. (2.9) has at least one positive solution $u \in C^{1}[0,1] \cap C(0,1)$ if $\lambda>0$ is chosen sufficiently small. 
Proof. To see this we will apply Theorem 2.1. Let $f(t, u)=\lambda a(t)\left(u^{\beta}-\frac{1}{u^{\alpha}}\right)$. Then $f:[0,1] \times(0,+\infty) \rightarrow(-\infty, \infty)$ is continuous and $\lim _{u \rightarrow 0^{+}} f(t, u)=-\infty$ uniformly on $[0,1]$.

In conditions $(H 5)$ and $(H 6)$ we let $a=c, b_{2}=2 c, b_{1}=0$. Also we let

$$
d_{0}=\max _{t \in[0,1]} a(t) \quad \text { and } \quad g_{1}(u)=\frac{\lambda d_{0}}{u^{\alpha}} \text { for } u \in(0, \infty)
$$

Then $g_{1}:(0, \infty) \rightarrow(0, \infty)$ is a continuous function and

$$
\begin{gathered}
\lambda a(t)\left(u^{\beta}-\frac{1}{u^{\alpha}}\right) \geq-g_{1}(u) \text { for } t \in[0,1], u>0 \\
\int_{0}^{a} g_{1}(s) d s=\int_{0}^{a} \frac{\lambda d_{0}}{s^{\alpha}} d s=\frac{\lambda d_{0}}{1-\alpha} a^{1-\alpha}<+\infty
\end{gathered}
$$

Also we have

$$
G_{1}(x)=\int_{0}^{x} \frac{\lambda d_{0}}{u^{\alpha}} d u=\frac{\lambda d_{0}}{1-\alpha} x^{1-\alpha}
$$

and

$$
\begin{aligned}
\int_{0}^{a}\left(G_{1}(x)\right)^{\frac{-1}{p}} d x & =\left(\frac{1-\alpha}{\lambda d_{0}}\right)^{\frac{1}{p}} \int_{0}^{a} x^{\frac{\alpha-1}{p}} d x \\
& =\left(\frac{1-\alpha}{\lambda d_{0}}\right)^{\frac{1}{p}} \cdot \frac{p}{\alpha+p+1} \cdot c^{\frac{\alpha+p+1}{p}}
\end{aligned}
$$

Then $\int_{0}^{a}\left(G_{1}(x)\right)^{\frac{-1}{p}} d x>q^{\frac{1}{p}}$, provided

$$
\lambda^{\frac{1}{p}}<\lambda^{*}=\left(\frac{1-\alpha}{q d_{0}}\right)^{\frac{1}{p}} \cdot \frac{p}{\alpha+p+1} \cdot c^{\frac{\alpha+p+1}{p}} .
$$

Next let

$$
g_{2}(u)=\lambda d_{0} u^{\beta} \text { for } u \in(0, \infty)
$$

Then $g_{2}:(0, \infty) \rightarrow(0, \infty)$ is a continuous function and

$$
\lambda a(t)\left(u^{\beta}-\frac{1}{u^{\alpha}}\right) \leq g_{2}(u) \text { for } t \in[0,1], u>0
$$

and

$$
G_{2}(x)=\int_{x}^{b_{2}} g_{2}(u) d u=\frac{\lambda d_{0}}{\beta+1}\left((2 c)^{\beta+1}-x^{\beta+1}\right) \text { for } x \in(0,2 c)
$$


Thus

$$
\begin{aligned}
\int_{b_{1}}^{b_{2}}\left(G_{2}(x)\right)^{-\frac{1}{p}} d x & =\int_{0}^{2 c}\left[\frac{\lambda d_{0}}{\beta+1}\left((2 c)^{\beta+1}-x^{\beta+1}\right)\right]^{-\frac{1}{p}} d x \\
& =\left(\frac{\beta+1}{\lambda d_{0}}\right)^{\frac{1}{p}} \int_{0}^{2 c} \frac{d x}{\left[(2 c)^{\beta+1}-x^{\beta+1}\right]^{\frac{1}{p}}} \\
& =\left(\frac{\beta+1}{\lambda d_{0}}\right)^{\frac{1}{p}} \cdot(2 c)^{\frac{p-\beta-1}{p}} \int_{0}^{1} \frac{d y}{\left[1-y^{\beta+1}\right]^{\frac{1}{p}}} \\
& \geq\left(\frac{\beta+1}{\lambda d_{0}}\right)^{\frac{1}{p}} \cdot(2 c)^{\frac{p-\beta-1}{p}} .
\end{aligned}
$$

Then $\int_{b_{1}}^{b_{2}}\left(G_{2}(x)\right)^{\frac{-1}{p}} d x>q^{\frac{1}{p}}$, provided with

$$
\lambda^{\frac{1}{p}}<\lambda^{* *}=\left(\frac{\beta+1}{q d_{0}}\right)^{\frac{1}{p}} \cdot(2 c)^{\frac{p-\beta-1}{p}} .
$$

Thus if $0<\lambda^{\frac{1}{p}}<\min \left\{\lambda^{*}, \lambda^{* *}\right\}$, the conditions of Theorem 2.1 are satisfied. As a result problem (2.9) has at least one positive solution.

\section{REFERENCES}

[1] R. ARIS, Introduction to the analysis of chemical reactors, Prentice-Hall, New Jersey, 1965.

[2] R. P. Agarwal, D. O'Regan, Nonlinear superlinear and nonsingular second order boundary value problems, J. Differential Equations, 143:1 (1998), pp. 60-95.

[3] K. LAN, J. R. L. WEBB, Positive solutions of semilinear differential equations with singularties, J. Differential Equations, 148:2 (1998), pp. 407-421.

[4] R. P. Agarwal, D. O'Regan, A note on existence of nonnegative solutions to singular semipositone problems, Nonlinear Analysis, 36:5 (1999), pp. 615-622.

[5] D. O'Regan, Some general existence principle and results for $\left(\phi\left(y^{\prime}\right)\right)^{\prime}=q f\left(t, y, y^{\prime}\right), 0<t<$ 1, SIAM J. Math. Anal., 24 (1993), pp. 648-668.

[6] H. LÜ AND D. O'Regan, A general existence theorem for the singular equation $\left(\varphi_{p}\left(u^{\prime}\right)\right)^{\prime}+$ $f(t, u)=0$, Math. Ineq. Appl., 5:1 (2002), pp. 69-78.

[7] Q. YAO AND H. Lü, Positive solutions of one-dimensional singular $p$-Laplace equations, Acta Mathematica Sinica, 41:6 (1998), pp. 1255-1264 (in Chinese).

[8] J. Cheng, Existence of positive solutions for a class of Dirichlet boundary value problem, Acta Mathematica Sinica, 45:2 (2002), pp. 301-306 (in Chinese).

[9] M. A. Del Pino, R. F. Manasevich and A. E. Murua, Existence and Multiplicity of Solutions with Prescribed Period For a Second Order Quasilinear O.D.E., Nonlinear Analysis, 18 (1992), pp. 79-92. 